\title{
Anabases
}

ANABASES Traditions et réceptions de l'Antiquité

$11 \mid 2010$

Varia

\section{La construction d'un mythe contemporain : les temples « sémitiques » d'Afrique romaine}

Meriem Sebaï

\section{OpenEdition}

1 Journals

Édition électronique

URL : http://journals.openedition.org/anabases/849

DOI : 10.4000/anabases.849

ISSN : 2256-9421

Éditeur

E.R.A.S.M.E.

Édition imprimée

Date de publication : 1 mars 2010

Pagination : 165-179

ISSN : 1774-4296

\section{Référence électronique}

Meriem Sebaï, « La construction d'un mythe contemporain : les temples « sémitiques » d'Afrique

romaine », Anabases [En ligne], 11 | 2010, mis en ligne le 01 mars 2013, consulté le 21 octobre 2019

URL : http://journals.openedition.org/anabases/849; DOI : 10.4000/anabases.849

(c) Anabases 
Anabases 11 (2010), p. 165-179.

\title{
La construction d'un mythe contemporain: les temples " sémitiques " d'Afrique romaine
}

Meriem Sebaï

Il est plus facile de dénoncer les erreurs, devenues patentes, de nos prédécesseurs, que d'éviter à notre tour de commettre de pareilles extrapolations, H. I. Marrou, De la connaissance historique, Paris, 1974, p. 145.

La connaissance est taillée sur le patron de documents mutilés [...], P. Veyne, Comment on écrit l'histoire, Paris, 1978, p. 26.

\begin{abstract}
À une éPOQue où les hommes POlitiQues réclament un débat sur les fondements identitaires de la France, il n'est pas inutile de s'interroger sur les constructions historiographiques issues de la pensée historique française. En revisitant l'histoire de l'élaboration du fait architectural complexe que constituent les temples dits sémitiques de l'Afrique romaine, une catégorie créée par les premiers explorateurs des territoires africains à la fin $\mathrm{du} \mathrm{XIX}{ }^{\mathrm{e}}$ siècle ${ }^{1}$, l'historien actuel ne peut qu'avoir présente à l'esprit cette phrase d'H.I. Marrou ou cette autre de P. Veyne. Elles semblent avoir été taillées sur mesure pour illustrer les difficultés dans lesquelles nous nous trouvons pour définir, appréhender et expliquer ce phénomène architectural qui jusqu'à nos jours bénéficie d'une exégèse directement issue du romantisme allemand et du courant philosophique français de l'histoire ${ }^{2}$, une exégèse fondée sur la recherche des origines orientales de l'Afri-

1 P. Carton, "Un édifice de Dougga en forme de temple phénicien", 1897, MSAF, p. $52-60$.

2 L'un de ses représentants, E. Renan, s'est attaché à traquer les " origines " des productions romaines qu'il étudiait : E. RenAn, De l'origine du langage, Paris, 1848; Histoire des origines du christianisme, Paris, 1863-1881.
\end{abstract}


que. Ce type d'approche et les présupposés idéologiques qu'il sous-tend fut pourtant remis en cause dès le milieu du XXe siècle par M. Bloch. Mais sa mise en garde contre le danger " hypnotique " que pouvait engendrer " l'idole des origines » est restée sans effet parmi l'historiographie d'après-guerre si bien que l'idée d'une civilisation de l'Afrique romaine jouissant d'un ancrage profond et vivace dans la matrice orientale par le biais de l'époque punique fut relayée et discutée avec enthousiasme, parfois de façon passionnée par plusieurs générations d'historiens spécialistes de l'Afrique antique ${ }^{3}$. Est-ce vrai? est-ce faux? Là n'est pas la question. Il n'est pas dans mon intention de construire mon propos sur une phénoménologie de l'erreur ou de l'exactitude ou encore de poser naïvement une série de questions nécessairement orientées: l'Afrique romaine assume-t-elle une identité pré-romaine ou sémitique forte ou tout simplement présente? Ou encore, les espaces du rituel dans une province donnée de l'empire romain véhiculent-ils une identité sémitique? Méconnaître d'emblée ces questions peut conduire à une réelle relecture de l'histoire religieuse de l'Afrique romaine. Se demander plutôt ce que nous apprennent ces temples dits sémitiques, décortiquer le processus de construction scientifique situé à la source du concept, réfléchir sur le bien-fondé de ce concept, conduit, en fait, à revenir sur le corpus documentaire.

Très vite, l'observation de cette catégorie particulière à l'Afrique romaine, permet de prendre conscience que nous ne pouvons traiter sereinement de l'histoire des temples de l'Afrique romaine, tout au plus pouvons-nous tirer quelques renseignements épars, à peine de quoi écrire une page sur ce que nous donnent à voir les documents. En l'occurrence, une documentation archéologique mal publiée et parfois irrémédiablement lacunaire et perdue. Cet aspect est important car une étude des choix architecturaux des Romains d'Afrique doit être conduite à partir d'une mise en contexte de la documentation et de l'" ambiance " dans laquelle se fait la recherche. Ce postulat méthodologique amène une première remarque. Choisir cette catégorie architecturale pour illustrer un dossier sur les erreurs des historiens conduit à se demander comment ce qui n'est qu'une hypothèse peut devenir en quelques années une certitude, puis en quelques décennies une évidence.

Quels sont les mécanismes scientifiques qui ont fait basculer cette catégorie du champ de l'hypothèse au statut de catégorie historique tangible?

Cette structure, également dénommée temple à cour ou temple romano-africain, apparaît durant l'époque romaine. Considérée depuis les prospections des explorateurs du XIX et du début du XXe siècle comme le dernier jalon de la participation des Africains à une forme de résistance religieuse à la romanisation, elle est au cœur d'un système de pensée basé sur l'idée que son processus planimétrique est issu de la sphère sémitique et participe, de ce fait, à la constitution d'une série de critères énonçant l'africanité

3 Pour une mise en perspective de ces débats: M. Sebaï, "La romanisation en Afrique, retour sur un débat. La résistance africaine: une approche libératrice?", Afrique et histoire, n 3 , 2005, p. 39-56. 
exacerbée des Africains de l'époque romaine qui, par le biais de la grande Carthage, se seraient définis comme les héritiers directs de $\mathrm{Tyr}^{4}$.

Cette conception est, à bien des égards, le lieu commun de la constitution d'un mythe contemporain, un mythe dont l'écriture a été tissée à l'aune d'une utilisation excessive des sciences historiques et entretenue par un discours idéologique émanant successivement d'esprits scientifiques en quête de légitimité identitaire ${ }^{5}$.

L'hypothèse de l'origo sémitique des temples de l'Afrique romaine à une ou trois cellae sans podium est fondée sur une équation simple proposée par les premiers explorateurs de l'Afrique antique pour permettre de distinguer ce qui est romain de ce qui est étranger à Rome. Ainsi, une fois que les critères énonçant les différences par rapport aux temples classiques de type italique, fondés sur le principe de la frontalité, construits sur un podium, furent définis, les archéologues isolèrent les caractéristiques d'édifices de conception très différente ${ }^{6}$. Or les critères spécifiques de reconnaissance de temples de type sémitique ou africain ou romano-africain sont les suivants: ils sont constitués de trois cellae ouvertes sur une cour entourée sur les côtés de portiques, le tout étant construit de plain-pied, sans podium, axialité et frontalité étant les emprunts faits aux temples italiques ${ }^{7}$. Cette structure aurait tiré son origine des aires sacrées à ciel ouvert, avec ou sans enceinte, d'époque punique que l'on a coutume d'appeler tophet ${ }^{8}$. A partir de la découverte des premiers vestiges de ce type à Thugga, Thuburnica, au Jebel Bou Kornine, les archéologues français, A. Merlin et L. Carton, entre autres, échafaudèrent le postulat que ces temples à cour et sans podium déclinaient nécessairement une structure antérieure à l'arrivée de Rome. Baptisés dès lors " temples africains d'origine sémitique ", ils permettaient d'établir que ce type de temple abritait un culte d'origine punique, ou romano-africain ou permettait d'induire une construction intellectuelle complexe sur l'africanité des dieux romains, guidant la recherche vers une lecture ethnique des divinités qui y étaient honorées 9 .

4 A. LÉzine, "Résistance à l'hellénisme de l'architecture religieuse de Carthage", Cahiers de Tunisie, n 26-27, 1959, p. 247-261.

5 Les pages d'introduction de l'ouvrage de M. BÉNABOU, La résistance africaine à la romanisation, Paris, 1976, p. 9-20, sont à cet égard éclairantes.

6 Ce dossier étant en cours de publication, je n'introduis ici qu'une bibliographie succincte relative à cette question: L. CARTON, "Le sanctuaire de Baal-Saturne à Dougga", NAM, VII, 1897, p. 367-474; A. Merlin, "Le temple d'Apollon à Bulla Regia", Notes et documents, I, 1908; L. CARTON, "Note sur la découverte d'un sanctuaire de Saturne dans la colonie de Thuburnica", CRAI, 1907, p. 380-384; L. CARTON, "Le sanctuaire de Baal-Saturne à Dougga”, NAM, VII, 1897, p. 367-474.

7 Reprenant le dossier des sanctuaires de Saturne en Afrique, M. LE GLAY, Saturne africain, Histoire, Paris, 1966, p. 275-282, tente de théoriser ce phénomène.

8 A. Lézine, Cah. Tun., 1959, p. 247-261.

9 C'est le postulat de M. Bénabou, Résistance, p. 331-375, lorsqu’il évoque l'africanisation des dieux romains en Afrique romaine. 
Par la suite, dans les années 1950, A. Lézine, architecte à la direction des Antiquités tunisiennes, intrigué par cette structure, établit en le théorisant un schéma évolutif des temples à cour sans podium selon l'ordre schématique suivant (fig. 1): un état primitif serait constitué d'un bétyle au centre d'une cour à portique. Durant une seconde étape, une cella est placée dans un nouvel enclos, puis le sanctuaire évolue encore par l'adjonction d'une, deux ou trois cellae, juxtaposées et projetées à l'extérieur du péribole primitif. Le nombre de cellae étant constitutif, selon une idée d'A. Merlin, du nombre de divinités honorées dans l'enceinte du lieu de culte et renvoyant aux dyades ou triades crées par l'esprit oriental ${ }^{10}$.

A
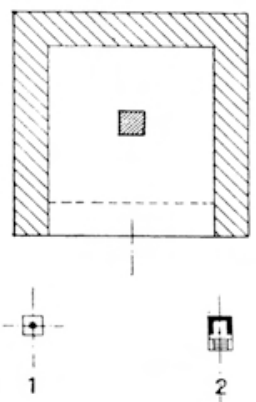

B

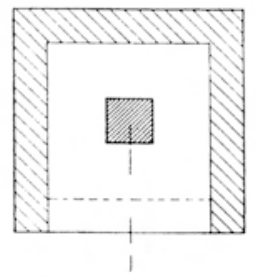

品
C

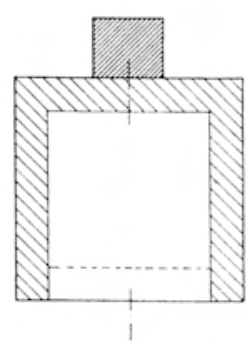

7
D

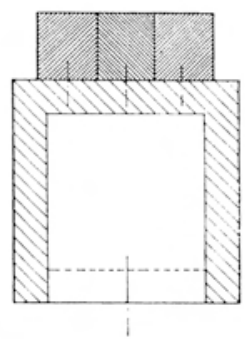

Fig. 1. Évolution des temples à cour en Afrique romaine.

A. Lézine, Cah. Tun., 1959, p. 259, fig. 4.

J'ai donc tenté, dans la mesure de mes moyens, d'aller jusqu'au bout de l'hypothèse de l'origine de ces temples d'Afrique romaine en interrogeant à la fois les sources planimétriques et archéologiques utilisées par ces deux premières générations d'historiens.

Comment fut élaboré le concept de temple dit sémitique? Et pourquoi le recours à cette catégorie est-il régulièrement défini par les historiens comme une donnée acquise, alors qu'une étude minutieuse des interprétations ne résiste pas à l'interrogation des sources. Pour tenter d'apporter une réponse possible, la mise en série de la documentation et l'observation des plans de temples constituera le fil conducteur de cette étude ${ }^{11}$.

10 A. Merlin, Apollon, p. 23.

11 Un travail plus ample sur l'ensemble de la documentation a été soutenu à l'université de Paris 1-Panthéon-Sorbonne: M. Sebaï, La vie religieuse dans les cités de Zeugitane $\left(\mathrm{I}^{\mathrm{er}}-\mathrm{IV}^{\mathrm{e}} \mathrm{s}\right.$. de n. è.). 
Fig. 2. Temple A de Kerkouane, fin $\mathrm{IV}^{\mathrm{e}}$ s. milieu III ${ }^{\mathrm{e}}$ s. av. n. è.

M'h. Fantar, Kerkouane, 1998, p. 56, pl. III.

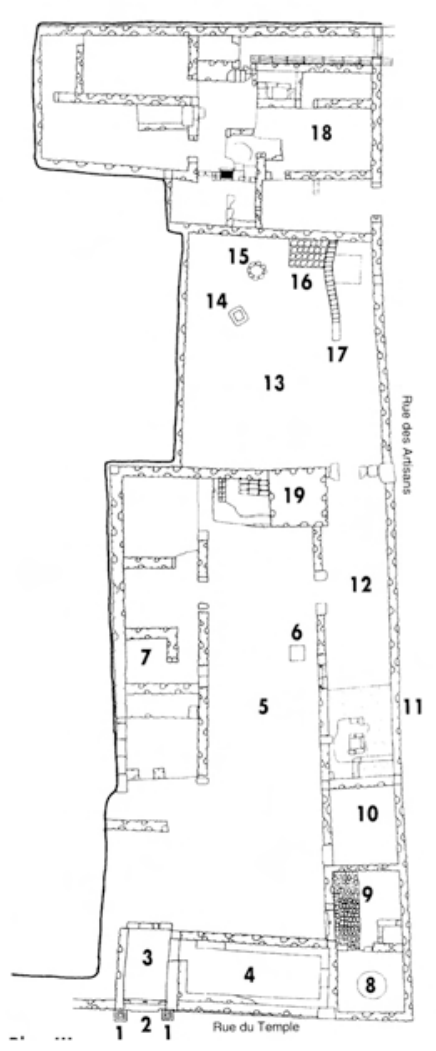

Lors de mes recherches dans l'historiographie africaniste, je n'ai rencontré ni réflexion ni questionnement sur les caractéristiques fondamentales du temple sémitique ni sur la pertinence du concept. Or en 1991, J. Margueron déplorait que malgré cent cinquante ans de fouilles au ProcheOrient, la connaissance du temple de type sémitique interdisait encore une approche synthétique, en raison du très petit nombre de certitudes ${ }^{12}$. D'autre part, pour répondre à la question: qu'est-ce qu'un "temple de type sémitique », il faudrait d'abord se demander ce qui relève du mot "sémitique ".

Plusieurs complications surgissent lorsque nous utilisons le terme "sémitique ". Faut-il l'employer dans son acception géographique ou ethnique? D'un point de vue géographique, ce terme ne s'applique pas exclusivement à la bande littorale où s'épanouissent les cités phéniciennes et, là encore, complexité et hétérogénéité sont les maîtres mots d'un contexte géographique, ethnique et politique diversifié. Car si des populations sémites sont installées dans cet espace immense ${ }^{13}$, elles ne sont pas les seules à l'avoir occupé et à avoir joué un rôle important dans le développement de l'univers religieux oriental ${ }^{14}$. Bien sûr, depuis l'Afrique, ce mot est utilisé dans son acception phénicienne en référence à la fondation tyrienne de Carthage, mais un tel terme renvoie avant tout à un groupe de langues étroitement apparentées, parlées par des populations qui ne sont pas forcément ethniquement homogènes ${ }^{15}$.

Informée du fait que les spécialistes de la recherche proche orientale estiment que les vestiges archéologiques dit " sanctuaires sémitiques " " ne peuvent que très rarement être attribués de façon spécifique à une tradition proprement sémitique de l'édifice

12 J. Margueron, "Sanctuaires sémitiques", Dictionnaire de la Bible, suppl. XI, 1991, col. 1104.

13 M. Dunand, "Phénicie", Dbibl., Suppl., VII, 1966, col. 1155-1204; G. Roux, La Mésopotamie. Essai d'histoire politique, économique et culturelle, Paris, 1985, p. 136-138.

14 Les Sumériens et les Hourrites sont ces autres populations qui cohabitent en même temps que les Sémites et que l'on distingue uniquement à partir de l'argument linguistique, cf. G. Roux, La Mésopotamie, p. 82-85 et p. 208-211.

15 G. Roux, La Mésopotamie, p. 240-241. 
religieux ${ }^{16}$ ", j’ai tenté de circonscrire le problème à partir des sources archéologiques découvertes sur le sol africain.

En quête de modèle, je me suis donc tournée vers deux sites ayant livré une documentation sans aucun doute d'époque punique. Les sites de Kerkouane et de Ras ed Drek dans le Cap Bon (fig. 2-3), apportent en première analyse, non pas un début de réponse mais, au contraire, une première série de questionnements auxquels il demeure difficile de répondre clairement, compte tenu du manque de précision des rapports de fouilles, de la complexité des contextes archéologiques et surtout de l'inachèvement de la fouille elle-même ${ }^{17}$. Si bien que l'on ne peut s'empêcher de douter à un moment ou à un autre de la nature des bâtiments que l'on a coutume d'intégrer dans le corpus des temples puniques. Le matériel mis au jour dans ces deux bâtiments ne permet pas de considérer que nous sommes en présence d'espaces religieux; en effet, l'indigence des restes organiques, l'absence de vaisselle, de dépôts votifs et de stèles qui sont en général les indicateurs courants de la présence d'un lieu de culte doivent inspirer la plus grande
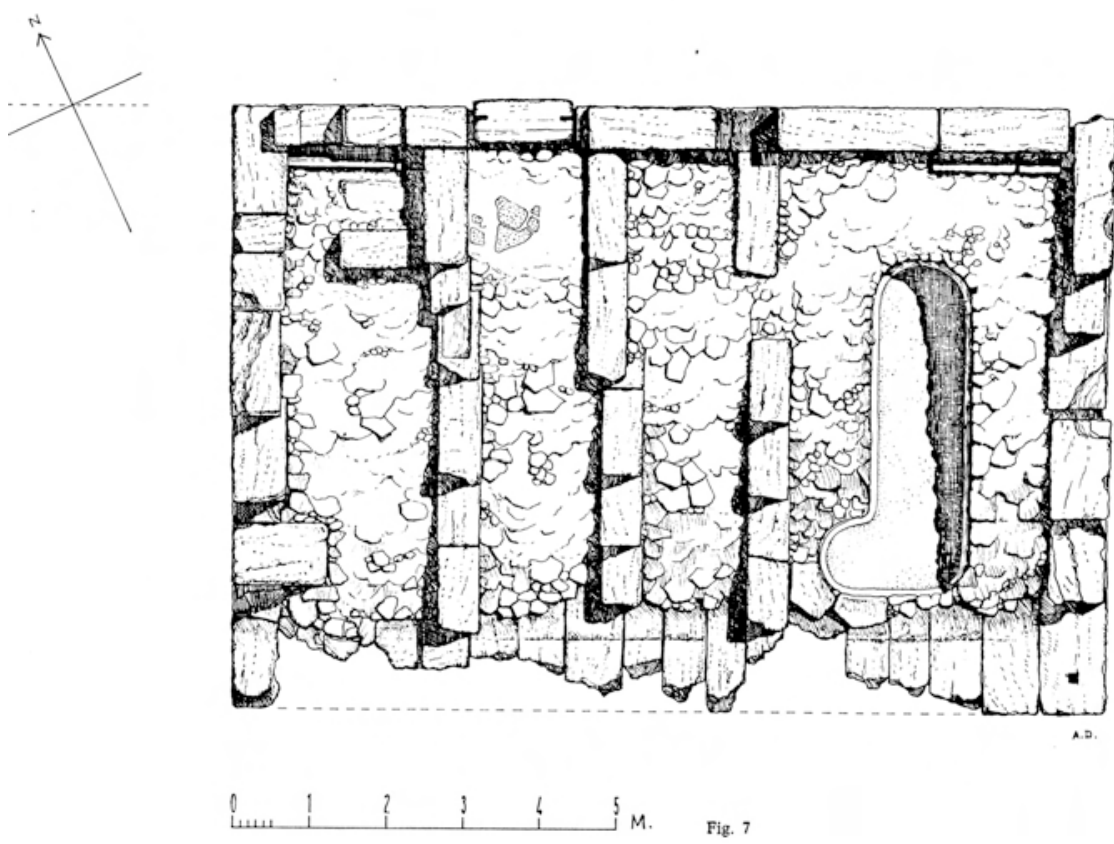

Fig. 3. Édifice de Ras ed Drek, F. Barreca, M’h. Fantar, Prospezione, 1983, II, fig. 7.

16 J. Margueron, Sanctuaires sémitiques, col. 1108-1109.

17 M. Fantar, Kerkouane, III, Tunis, 1986, p. 147-221; M’h. Fantar, "Le temple de Ras ed Drek”, F. Barreca, M’h. Fantar, (éd.), Prospezione archeologica al Capo Bon, II, 1983, p. 43-63. 


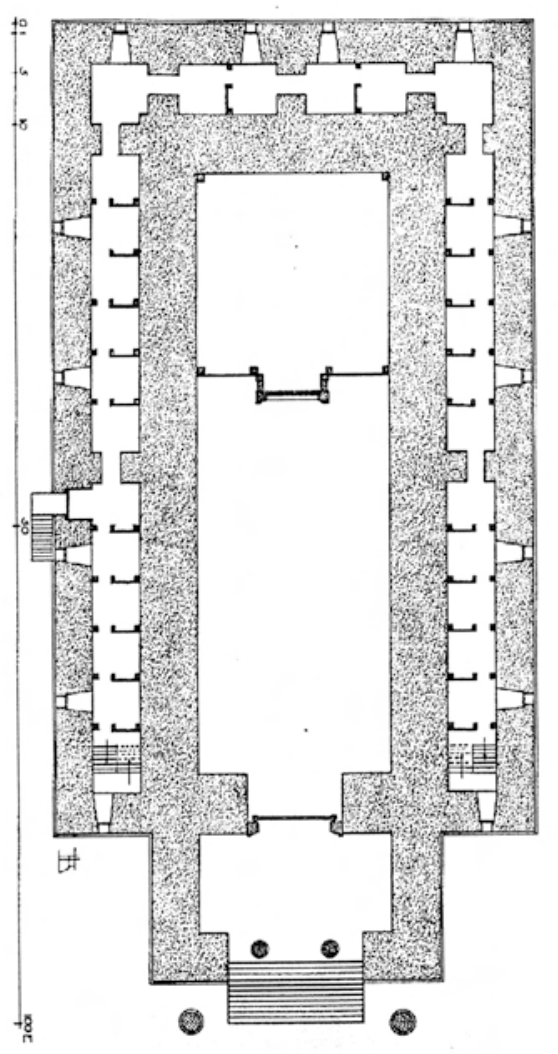

Fig. 4 . Reconstitution du temple de Jérusalem, Th. Busink, Der Tempel, 1970, pl. 48. prudence. Ces deux édifices sont pourtant intégrés dans l'architecture sacrée et englobés dans la filiation du temple de Jérusalem ${ }^{18}$.

J'ai donc suivi tout naturellement la piste indiquée par l'historiographie africaniste, celle du temple de Salomon, à Jérusalem (fig. 4). En l'absence de faits archéologiques, cette hypothèse m'est toujours apparue parfaitement incongrue et, malgré ce que nous révèle l'Ancien Testament, la recherche de l'archétype du temple punique semble de toutes les manières vouée à l'échec ${ }^{19}$. Affirmer, comme le fit A. Lézine ou d'autres après lui ${ }^{20}$, que le temple de Jérusalem, en raison de ses concepteurs phéniciens, serait en quelque sorte le premier temple phénicien, ne doit pas nous faire oublier que, pour séduisante qu'elle soit, cette hypothèse ne repose que sur la virtualité d'un édifice auquel sa forte valeur symbolique a conféré un statut de modèle, sans qu'on en possède la moindre trace archéologique. Cette hypothèse archétypale se heurte à trop de précautions d'ordre méthodologique pour pouvoir être considérée avec sérieux. Elle induit avec trop de certitude la question de la dimension historique de l'Ancien Testament.

Si l'on opère un retour sur la documentation africaine, on constate que malgré les difficultés exposées, toute l'argumentation sur un paradigme phénicien est approfondie par les trois premières générations de chercheurs travaillant en Afrique du Nord. L'architecte A. Lézine, l'archéologue G. Ch.-Picard et l'historien M. Le Glay, ont repris des hypothèses de L. Carton et A. Merlin émises au moment de la découverte de ces édifices religieux.

18 M'H. FanTAR, Kerkouane, III, p. 174.

19 I Rois, 6-7; II Chroniques, 3-4.; Th. A. Busink, Der Tempel von Jerusalem von Salomo bis Herodes, Leyde, T. I, 1970, T. II, 1980.

20 S. Moscati, I Fenici e Carthaginesi in Sardegna, Milan, 1968, p. 100-114; F. Barreca, Monte Sirai, I, p. 32-33. 


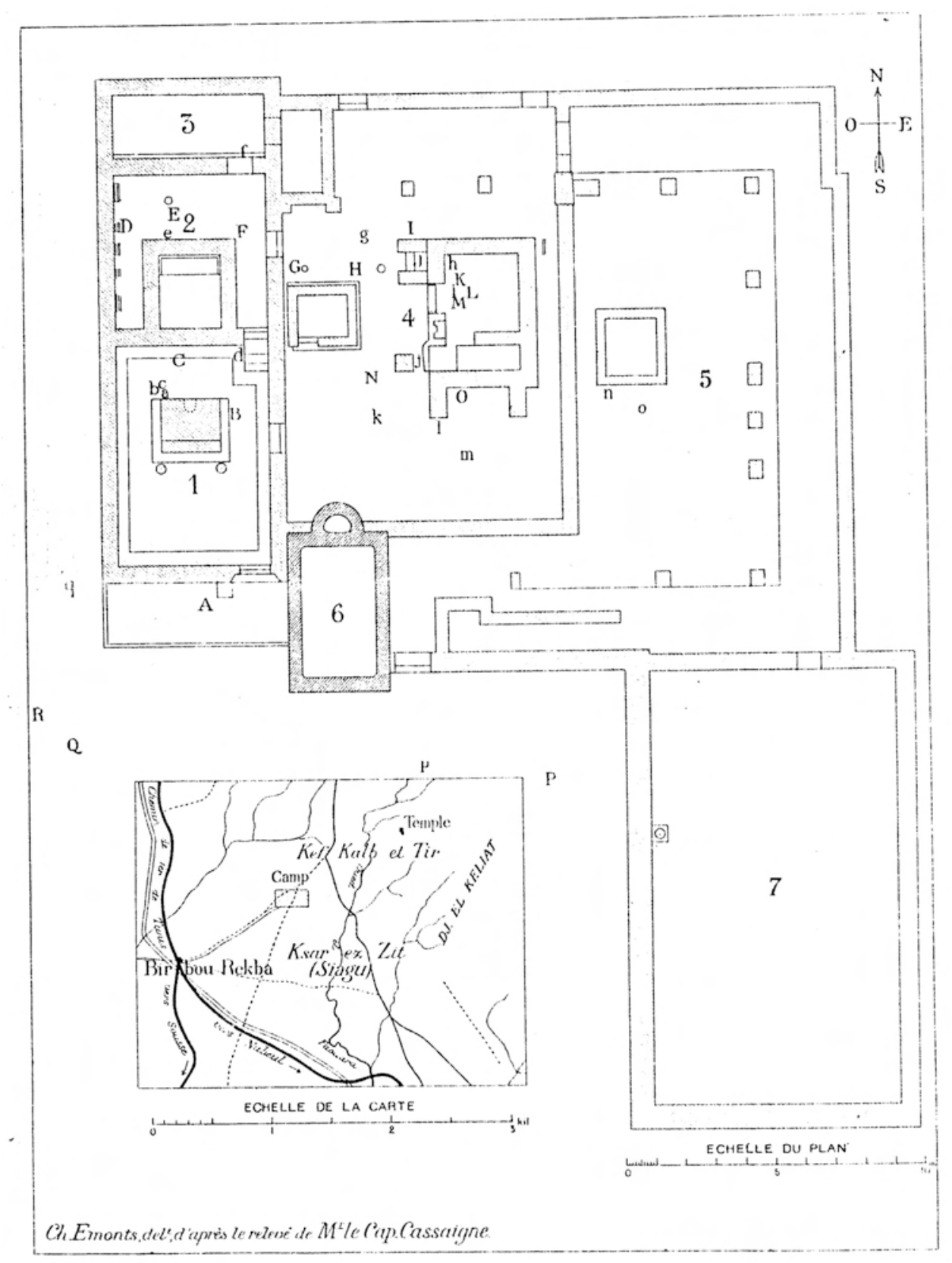

Fig. 5. Plan du sanctuaire de Thinissut, II $^{\mathrm{e}}$ s. av. n. è.-II ${ }^{\mathrm{e}}$ s. de n. è., A. Merlin, $N \ll D$, 1910, fig. ${ }^{\circ} 1$. 
A. Lézine, qui proposa de théoriser un processus évolutif de ces édifices sans aucune documentation chronologique tangible, crée de toute pièce une situation idéale. Sur le terrain, les situations sont diverses et beaucoup plus complexes, et dans l'écrasante majorité des cas, la restitution de Lézine est impossible à vérifier.

À l'origine de sa théorie, on trouve deux édifices dégagés au début du siècle: le sanctuaire de Thinissut ${ }^{21}$ (fig. 5) et celui d'El Kenissia ${ }^{22}$ (fig. 6). Dans un article co-écrit avec $\mathrm{H}$. Dridi, nous avons démontré que la complexité des structures planimétriques due aux réductions et aux redéploiements successifs qu'a subis ce lieu de culte montre que la recherche d'un archétype planimétrique oriental est vaine ${ }^{23}$.

À El Kenissia, l'ensemble des premières constatations qui se dégagent de l'observation du plan de ce sanctuaire relève de l'irrégularité, de la dissymétrie et de la distorsion de l'orientation des structures internes. Cette organisation est due au fait que, tout comme à Thinissut, ce bâtiment a connu plusieurs phases de réalisation dont il est difficile de reconnaître la logique. Mais Lézine pensait pouvoir reconnaître une logique liée d'une part au temple de Jérusalem et d'autre part à l'étude de l'évolution du temple de Byblos $^{24}$ situé entre le III $^{\mathrm{e}}$ millénaire av. n. è. et le XVIII siècle av. n. è. (fig. 7-8). Il n'est pas inutile de citer un des passages concernant le temple de Byblos: «Il y a là des temples très anciens, qui comportent une ou plusieurs cellae disposées au fond d'une cour. Il n'est pas besoin d'aller chercher ailleurs l'origine des temples sans podium de Thinissut, d'El Kenissia, Bulla Regia, ou Thugga. Loin d'être redevables à l'influence romaine, ces sanctuaires constituent, au contraire, des témoignages supplémentaires de l'attachement des Puniques aux traditions de leur mère patrie ${ }^{25}$." De fait, le modèle giblite qu'utilise Lézine n'est pas performant, car le bétyle entouré d'une chapelle donnant naissance à trois chapelles est inversé, l'absence de portique incite à la prudence et l'écart chronologique entre le dernier état du temple de Byblos et les temples d'Afrique romaine est vertigineux. Peut-on alors envisager avec objectivité une relation évolutionniste entre ces "représentants » de la planimétrie religieuse orientale des $\mathrm{III}^{\mathrm{e}}$ et II ${ }^{\mathrm{e}}$ millénaires et ceux de l'Afrique romaine? Il parait clair que Lézine et ses successeurs cherchaient à les intégrer dans le moule des persistances pré-romaines et, par-delà, à les faire remonter à la mère patrie orientale, mais la filiation établie entre le temple de Jérusalem, Byblos, Thinissut et el Kenissia se démantèle d'elle-même lorsque l'on y regarde de plus près. Ainsi, loin

21 A. Merlin, "Le sanctuaire de Baal et de Tanit près de Siagu", Notes et Documents, IV, 1910.

22 L. Carton, "Le sanctuaire de Tanit à El Kenissia", Mémoire de l'Académie des inscriptions et Belles Lettres, XII, Paris, 1908.

23 M. Sebaï, H. Dridi, "De Tanesmat à Thinissut. Nouvelles observations sur l'aménagement d'un lieu culte africain", Lieux de cultes: aires votives, temples, églises, mosquées, IX colloque international sur l'histoire et l'archéologie de l'Afrique du Nord antique et médiévale, Tripoli, 2005, p. 101-117.

24 M. Dunand, Fouilles de Byblos, Paris, 1937-1958.

25 A. Lézine, Cah. Tun., 1959, p. 256. 


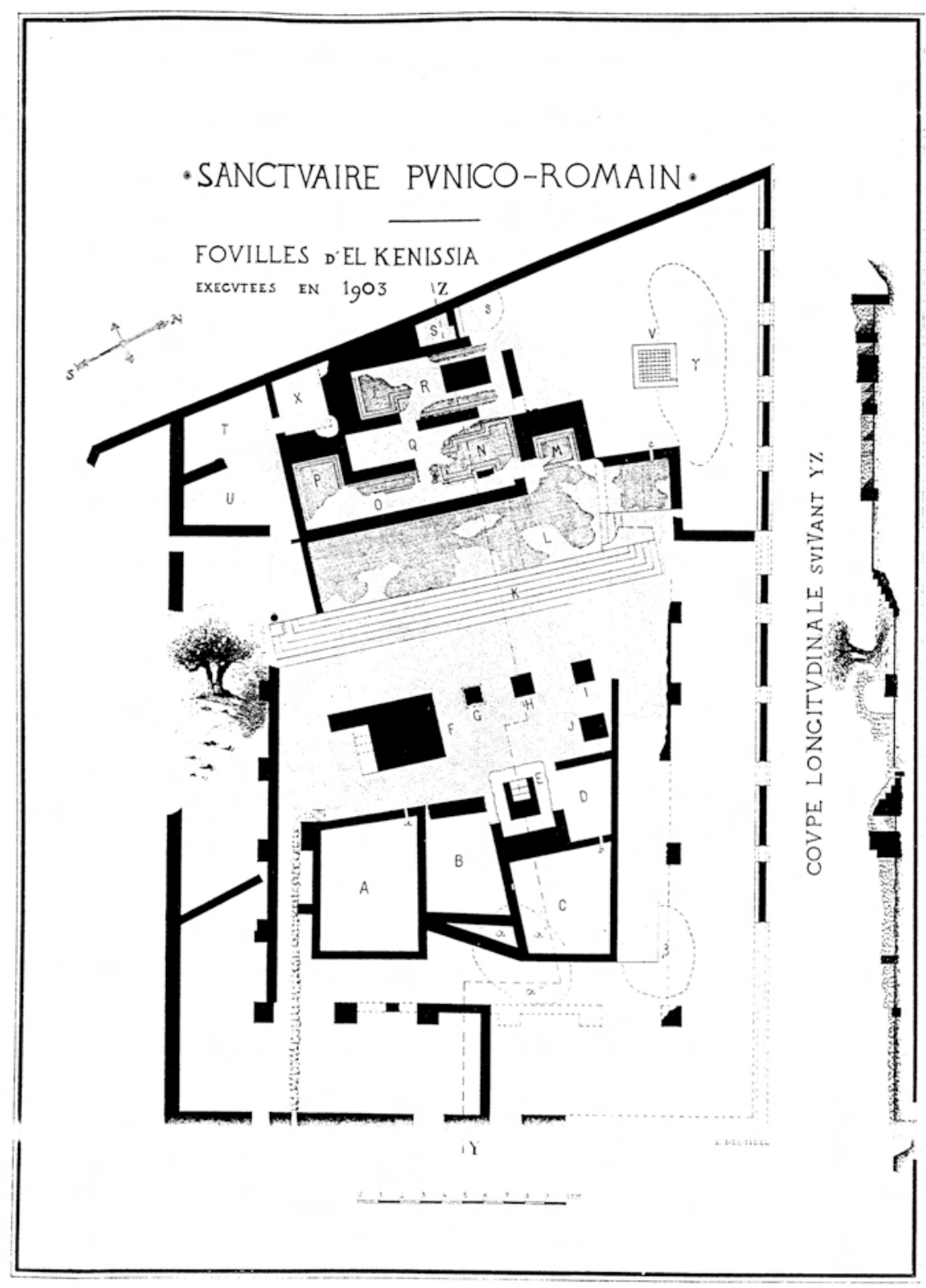

Fig. 6. Sanctuaire de El Kenissia,

L. Carton, El Kenissia, 1906, pl. 1. 


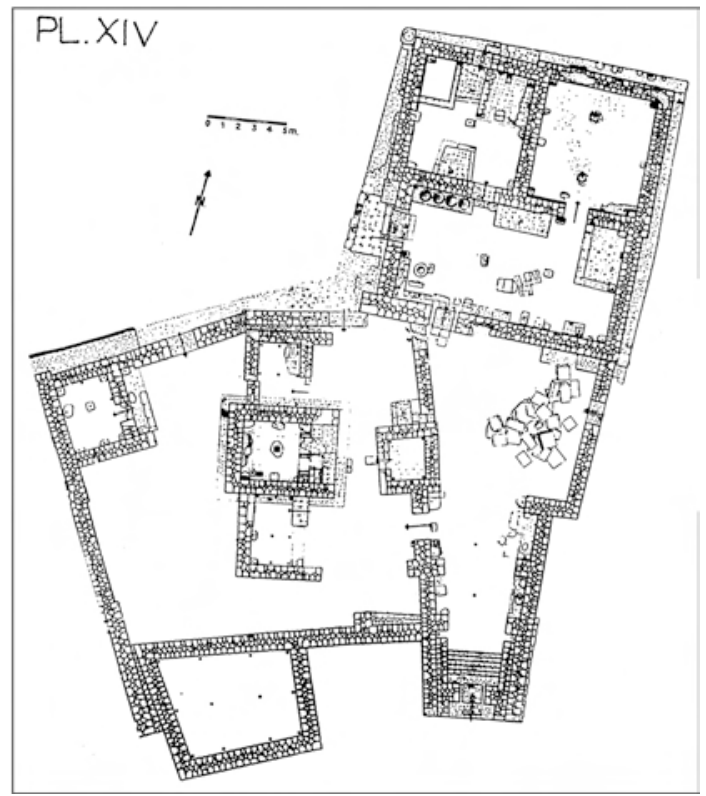

Fig. 7. Temple de Reschef à Byblos, $3^{\mathrm{e}}$ millénaire av. n. è.,

Dictionnaire de la Bible, 1991, n 967.

d'apporter des solutions de continuité avec une matrice sémitique, les sources archéologiques semblent indiquer des solutions de discontinuité et de rupture avec un paradigme qui, de toute évidence fait défaut. A. Lézine a construit une théorie des " temples africains de tradition sémitique " à partir du temple de Jérusalem, édifice marquant l'imaginaire dont on ne pourra jamais découvrir les structures archéologiques originelles.

Tournons-nous maintenant vers une localité romaine d'Afrique proconsulaire, la colonie honoraire de Bulla Regia, où un temple répondant aux critères institués par $\mathrm{A}$. Lezine a été mis au jour.

Dans cette cité, les dégagements qui ont eu lieu en 1905 ont contribué à mettre au jour, en même temps qu'un édifice religieux, une série de quatre inscriptions permettant d'identifier le monument comme un temple d'Apollon Auguste ${ }^{26}$. Cet édifice, construit de plain-pied avec la cour du forum, est composé d'une cour à portique s'ouvrant sur une cella centrale au nord. Une seconde ainsi qu'une troisième pièces, situées respectivement à l'ouest et à l'est ont été mises en évidence. A. Merlin cru pouvoir identifier cet ensemble à un temple à trois cellae de type sémitique (fig. 9) abritant une triade préromaine. De cette interprétation du monument est né un ensemble d'hypothèses sur l'identité des divinités vénérées dans le temple. Les documents mentionnant Apollon, le Genius coloniae Bullensium Regiorum et les dieux augustes ${ }^{27}$, A. Merlin et tous les historiens qui se sont intéressés à cet édifice ont bâti une histoire de la vie religieuse de la cité basée sur l'indigénité des divinités, anciens dieux de la cité numide qui se seraient perpétués durant l'Empire sous un vernis de romanité ${ }^{28}$. Or, une simple observation, in situ, des structures et des enduits

26 A. Merlin, Apollon, p. 6-27.

27 C. $25511(A E, 1906,140)$; C. $25513(A E, 1907,22 b)$; C. 25512 (AE, 1907, 22a); C. 25510.

28 J. ToutAin, Les cultes païens dans l'Empire romain. Les cultes indigènes, nationaux et locaux. Les cultes Ibériques, $2^{\mathrm{e}}$ fascicule, III, Paris, 1917, p. 1-2; G.-Ch. PICARD, Les religions de 
utilisés pour la construction des différentes cellae permet d'établir que les deux espaces situés à l'est et à l'ouest de la cella centrale ont été ajoutés à une époque ultérieure à la construction du temple et qu'elles ne font pas partie du programme initial. Par conséquent, cet édifice était probablement à l'origine composé d'une cella axiale. Les salles latérales ne témoignent pas d'activités rituelles. Avec S. Saint-Amans, nous avons montré par le croisement des sources iconographiques, épigraphiques et archéologiques que la communauté divine accompagnant Apollon Auguste dans ce temple avait été définie par l'ordo local au moment de l'érection du municipe au rang de colonie et que nous étions donc face à des divinités romaines fondatrices de la nouvelle identité juridique de la cité ${ }^{29}$.

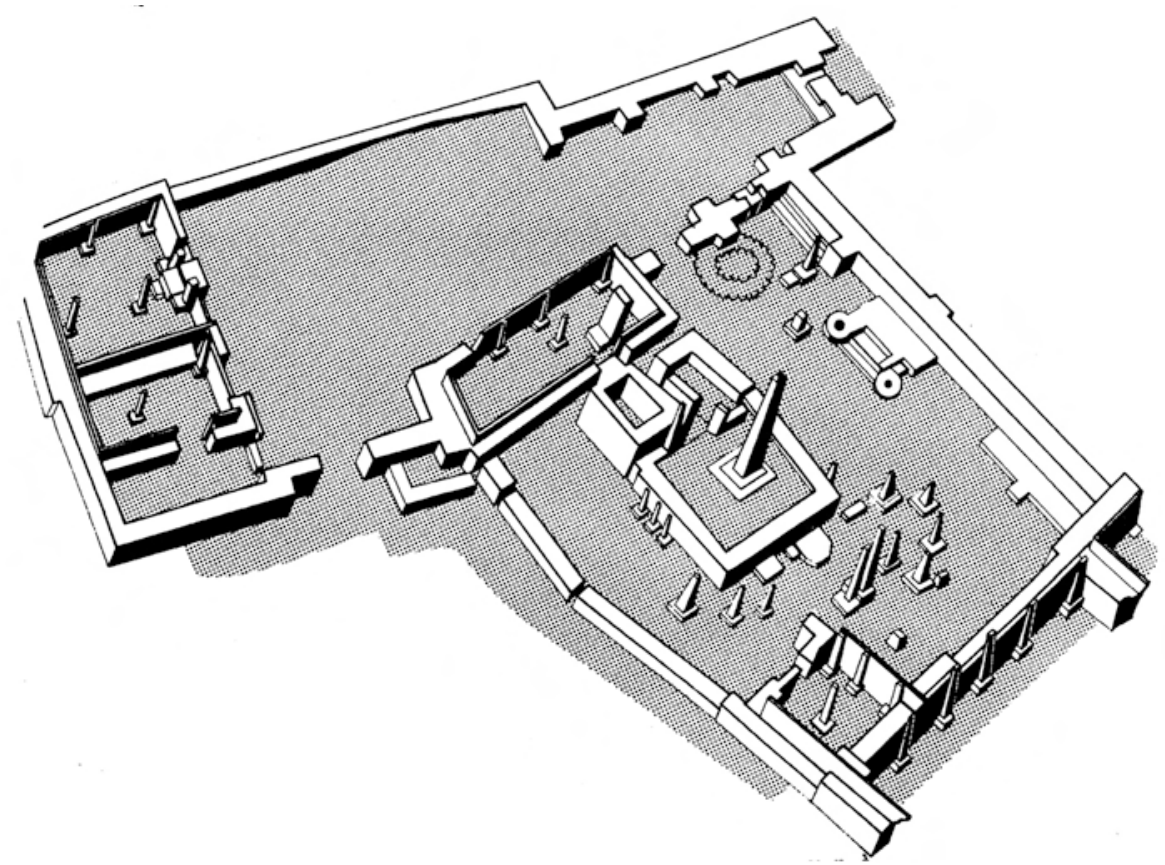

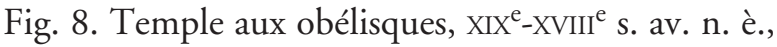

P. Amiet, Proche-Orient, 1977, pl. 847.

l'Afrique antique, Paris, 1954, p. 158.

29 S. Saint-Amans, M. Sebaï, "Genius en Afrique en romaine: dossiers iconographiques et épigraphiques. Nouvelles observations sur la représentation et la définition d'une divinité romaine", $1^{e r}$ colloque sur l'iconographie du Maghreb antique, Tunis, février 2008 (sous presse) 


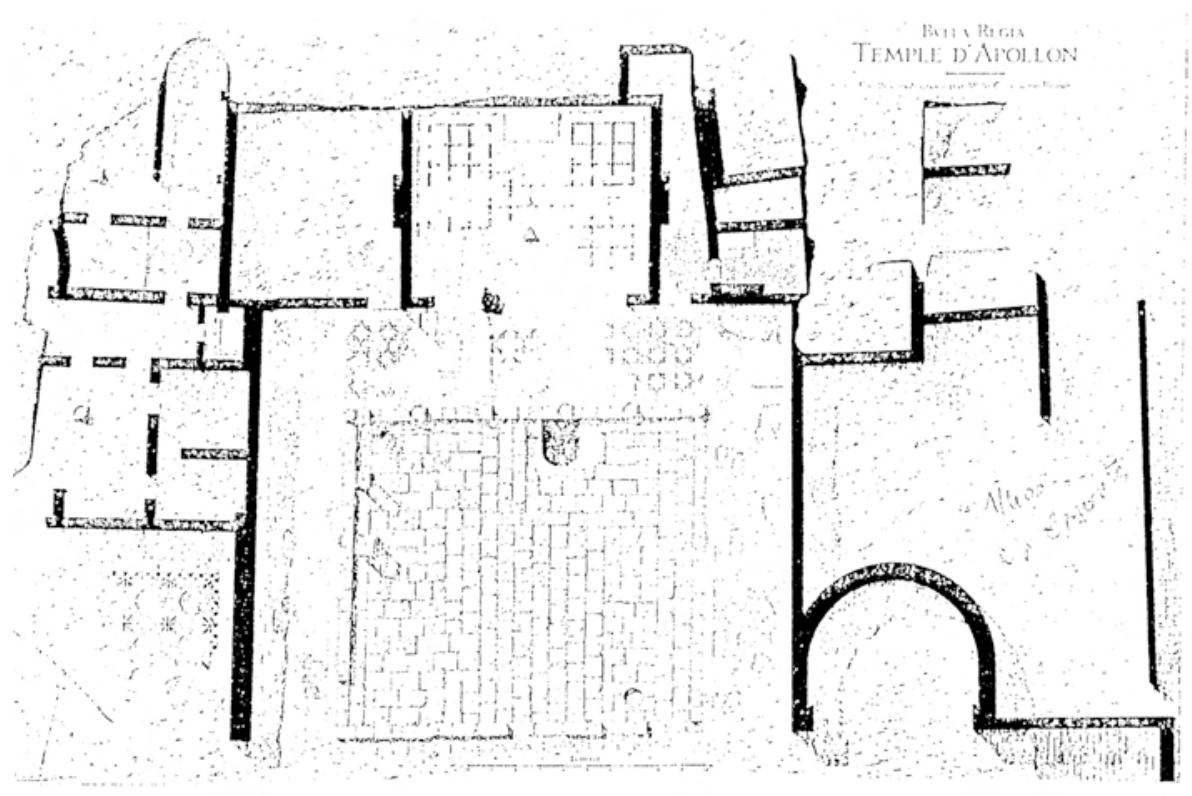

Fig. 9. Temple d'Apollon à Bulla Regia, 128 de n. è., A. Merlin, $N \mathcal{} \mho D, 1908$, p. 7, fig. 1.

En définitive, peut-on parler d'un type de temple sémitique à trois cellae, à cour et sans podium dont l'évolution dans le temps et l'espace se manifesterait à travers les temples d'El Kenissia et de Thinissut durant la période punico-romaine, puis qui s'étendrait à l'époque romaine à des temples tels que ceux d'Apollon à Bulla Regia? Le temple de Salomon et sa tripartition verticale restituée d'un côté, de même que celui de Byblos et sa tripartition horizontale de l'autre, ne sont pas, ne peuvent pas être les modèles lointains des temples à trois cellae d'époque romaine, il suffit pour s'en convaincre d'analyser et d'observer la planimétrie et les structures du bâti des édifices orientaux et occidentaux. Cette typologie architecturale peut maintenant être abandonnée, comme peuvent l'être également le schéma évolutif et les thèses de l'origo qui y sont attachés. Ce constat ne signifie pas pour autant qu'il faille nier la problématique des persistances. Non. Il faut tout " naturellement » la replacer dans un contexte débarrassé de tout postulat idéologique et de tout étiquetage nationaliste.

Si la formule de H.I. Marrou, mise en exergue de ces quelques feuillets, résonne avec force au moment de conclure, les réflexions de M. Bloch sur la notion des origines conduisent à préciser encore mon propos: «[...] les origines, écrit-il, sont un commencement qui explique. Pis encore: qui suffit à expliquer. Là est l'ambiguïté; là est le danger ${ }^{30}$." 
Il semble que les temples de Thinissut, d'El Kenissia, d'Apollon à Bulla Regia, ainsi que beaucoup d'autres lieux de culte dits de type "sémitique " - non abordés dans ce cadre - ont participé d'une vision mythique très tenace dans l'historiographie africaniste qui place l'Afrique romaine dans une tradition archétypale orientale. Les contextes archéologiques qui nous ont été transmis révèlent plutôt un phénomène complexe qui ne s'avère pas avoir été rattaché à un modèle archaïque ou classique. Ce que l'on décèle d'après l'énoncé des sources indique plutôt une multitude d'éléments planimétriques dont le dénominateur commun est l'absence de podium, la présence d'une cella et d'un portique centrés sur un autel. Cette formule structurelle, se déclinant différemment selon les cités, associée à la richesse épigraphique et iconographique que recèlent ces temples permet d'émettre l'hypothèse que ces éléments une fois replacés dans leur contexte poliade déclinent une identité en conformité avec le modèle romain.

Les contextes politiques contemporains dans lesquels est né et s'est épanoui ce mythe nous permettent de comprendre pourquoi la catégorie des temples dits sémitiques a eu un écho considérable tant à l'époque coloniale qu'à celle des nationalismes maghrébins. Elle relève de l'affirmation et de la réaffirmation idéologique d'une quête de pureté originelle, fantasmée et déclinée de multiples façons par les premiers prospecteurs de l'Afrique antique, qui tentaient de rattacher à un modèle pur des structures qui leur étaient étrangères ${ }^{31}$. A notre époque, la question des origines des temples sémitiques est encore d'actualité. On distingue derrière cette question un projet plus ou moins conscient, plus ou moins avoué, de rattacher à une culture " primordiale " ou très ancienne et par là même "plus légitime ", l'architecture sacrée de l'Afrique romaine pour lui trouver une ramification conduisant à un concept religieux unique.

Cette conception tenace d'une culture occidentale punique immuablement liée à la mère patrie empêche de considérer les réélaborations occidentales dans leurs acceptions régionales et dans leur dissonance avec un schéma oriental. Peut-être est-il temps, maintenant, en opérant un retour sur la documentation de replacer l'Afrique dans son contexte spatial et temporel, celui d'une province romaine dans laquelle les communautés poliades réalisèrent un certain nombre de modifications conscientes et volontaires qui leur permirent d'intégrer un univers en pleine mutation.

Meriem SeBAï

MCF Paris I - Panthéon Sorbonne - UMR 8585

meriemsebai@gmail.com

31 Il s'agissait de créer pour ce qui semblait " autre » une matrice équivalente aussi prestigieuse que Rome ou l'Italie, Tyr ou la Phénicie. 


\section{Bibliographie}

BARreCA, F. Ricerche puniche a Monte Sirai, I, Rapporto preliminare della missione archeologica dell'Università di Roma e della Soprintendenza alle Antichità di Cagliari, Rome, 1964.

BÉNABOU, M. La résistance africaine à la romanisation, Paris, 1976.

Bloch, M. Apologie pour l'histoire ou Métier d'historien, Paris, 1997.

Busink, Th. A. Der Tempel von Jerusalem von Salomo bis Herodes, Leyde, t. I, 1970, t. II, 1980.

Carton, P. "Un édifice de Dougga en forme de temple phénicien ", 1897, MSAF, p. 52-60.

Carton, L. "Le sanctuaire de Tanit à El Kenissia ", Mémoire de l'Académie des inscriptions et Belles Lettres, XII, Paris, 1908.

Dridi, H. ; Sebaï, M. "De Tanesmat à Thinissut. Nouvelles observations sur l'aménagement d'un lieu culte africain ", Lieux de cultes: aires votives, temples, églises, mosquées, IX colloque international sur l'histoire et l'archéologie de l'Afrique du Nord antique et médiévale, Tripoli, 2005, p. 101-117.

Dunand, M. Fouilles de Byblos, Paris, 1937-1958.

Dunand, M. "Phénicie ", Dictionnaire de la bible, suppl., VII, 1966, col. 1155-1204.

Fantar, M'H. Kerkouane, cité punique du Cap Bon (Tunisie), III, Tunis, 1986.

Le Glay, M. Saturne africain, Histoire-Monuments, I-II, Paris, 1966.

Margueron, J. "Sanctuaires sémitiques ", Dictionnaire de la bible, suppl. XI, 1991, col. 1104.

Merlin, A. "Le temple d'Apollon à Bulla Regia », Notes et documents, I, 1908.

Merlin, A. "Le sanctuaire de Baal et de Tanit près de Siagu ", Notes et Documents, IV, 1910.

Moscati, S. I Fenici e Carthaginesi in Sardegna, Milan, 1968.

PICARD, G.-Ch. Les religions de l'Afrique antique, Paris, 1954.

Renan, E. De l'origine du langage, Paris, 1848.

RENAN, E. Histoire des origines du christianisme, Paris, 1863-1881.

Roux, G. La Mésopotamie. Essai d'histoire politique, économique et culturelle, Paris, 1985, p. 136-138.

Saint-Amans, S.; Sebaï, M. "Genius en Afrique en romaine: dossiers iconographiques et épigraphiques. Nouvelles observations sur la représentation et la définition d'une divinité romaine ", $1^{e r}$ colloque sur l'iconographie du Maghreb antique, Tunis, février 2008 (sous presse).

SEBAÏ, M. "La romanisation en Afrique, retour sur un débat. La résistance africaine: une approche libératrice? ", Afrique et histoire, n 3, 2005, p. 39-56.

TouTAIN, J. Les cultes païens dans l'Empire romain. Les cultes indigènes, nationaux et locaux. Les cultes Ibériques, $2^{\mathrm{e}}$ fascicule, III, Paris, 1917.

Veyne, P. Comment on écrit l'histoire, Paris, 1978. 